\title{
GLOBAL LIMITING EMBEDDINGS OF LOGARITHMIC BESSEL POTENTIAL SPACES
}

\author{
PETR GURKA AND BOHUMÍR OPIC
}

\begin{abstract}
The paper is a continuation of [EGO II-IV], where it was shown that Bessel potential spaces $H^{\sigma} Y\left(\mathbb{R}^{n}\right)$, modelled upon appropriate generalized Lorentz-Zygmund spaces $Y\left(\mathbb{R}^{n}\right)$ may be embedded into Orlicz spaces $L_{\Phi}(\Omega)$, where $\left.\Phi(t)=\exp \left(\exp \left(\ldots \exp t^{v}\right) \ldots\right)\right)$ for large $t, v>0$, and $\Omega$ is a subset of $\mathbb{R}^{n}$ with finite volume. Using weighted Hardy inequalities, we modify the Young function $\Phi$ near the origin so that the above embedding holds with $\Omega$ replaced by $\mathbb{R}^{n}$. The resulting Young function dominates globally the Young function $\Psi(t)=t^{q}, t>0$, for $q$ sufficiently large and consequently, $H^{\sigma} Y\left(\mathbb{R}^{n}\right) \hookrightarrow L^{q}\left(\mathbb{R}^{n}\right)$. We also obtain an estimate of the norms of the last embeddings which is sharp in their dependence upon $q$ provided that $q$ is large enough.
\end{abstract}

Mathematics subject classification (1991): 46E35, 46E30, 26D15.

Key words and phrases: Generalized Lorentz-Zygmund spaces, logarithmic Bessel potential spaces, Orlicz spaces, embeddings.

\section{REFERENCES}

[A] R. A. AdAms, Sobolev spaces, Academic Press, New York, 1975.

[AS] N. ARonszajn And K. T. Smith, Theory of Bessel potentials, Part I, Ann. Inst. Fourier 11 (1961), 385-475.

[BR] C. BEnNett And K. Rudnick, On Lorentz-Zygmund spaces, Dissertationes Math. 175 (1980), $1-72$.

[BS] C. Bennett AND R. Sharpley, Interpolation of operators, Pure Appl. Math. 129, Academic Press, New York, 1988.

[BW] H. BRÉZIS AND S. WAINGER, A note on limiting cases of Sobolev embeddings and convolution inequalities, Comm. Partial Differential Equations 5 (1980), 773-789.

[EE] D. E. Edmunds AND W. D. Evans, Orlicz and Sobolev spaces on unbounded domains, Proc. Roy. Soc. London Ser. A 342 (1975), 373-400.

[EGO I] D. E. EDMunds, P. GURKA AND B. OPIC, Double exponential integrability of convolution operators in generalized Lorentz-Zygmund spaces, Indiana Univ. Math. J. 44 (1995), 19-43.

[EGO II] D. E. EDMUnDS, P. GURKA AND B. OPIC, Double exponential integrability, Bessel potentials and embedding theorems, Studia Math. 115 (1995), 151-181.

[EGO III] D. E. EDMUNDS, P. GURKA AND B. OPIC, Sharpness of embeddings in logarithmic Bessel-potential spaces, Proc. Roy. Soc. Edinburgh 126A (1996), 995-1009.

[EGO IV] D. E. EDMUNDS, P. GURKA AND B. OPIC, On embeddings of logarithmic Bessel potential spaces, J. Functional Anal. 146 (1997), 116-150.

[EGO V] D. E. EDMUNDS, P. GURKA AND B. OPIC, Norms of embeddings of logarithmic Bessel potential spaces, Proc. Amer. Math. Soc. (to appear).

[EK] D. E. Edmunds And M. Krbec, Two limiting cases of Sobolev imbeddings, Houston J. Math. 21 (1995), 119-128.

[EOP] W. D. EvANS, B. OPIC AND L. PICK, Interpolation of operators on scales of generalized Lorentz-Zygmund spaces, Math. Nachr. 182 (1996), 127-181. 
[FLS] N. Fusco, P. L. Lions And C. SBordone, Sobolev imbedding theorems in borderline cases, Proc. Amer. Math. Soc. 124 no. 2 (1996), 561-565.

[O] R. O'NeIL, Convolution operators and L $(p, q)$ spaces, Duke Math. J. 30 (1963), 129-142.

[OK] B. OPIC AND A. KufNER, Hardy-Type Inequalities, Pitman Research Notes in Mathematics 219, Longman, Harlow, 1990.

[OP] B. OPIC AND L. PICK, On generalized Lorentz-Zygmund spaces (to appear).

[T] N. S. TRUDINGER, On imbeddings into Orlicz spaces and some applications, J. Math. Mech. 17 (1967), 473-484.

[Z] W. ZIEMER, Weakly differentiable functions, Graduate Texts in Math. 120, Springer, Berlin, 1989. 